\title{
Abstracting s-semantics Using A Model-Theoretic Approach
}

\author{
Dmitri Boulanger Maurice Bruynooghe $^{\star \star} \quad$ Marc Denecker $^{\star \star \star}$ \\ Department of Computer Science \\ Katholieke Universiteit Leuven \\ Celestijnenlaan 200 A, B-3001, Heverlee, Belgium \\ email: \{dmitri, maurice, marcd\}@cs.kuleuven.ac.be
}

\begin{abstract}
A new approach for abstract interpretation of definite logic programs is introduced. The basic idea is to use an equality theory which safely approximates the Free Equality theory. The non-Herbrand model generated from this theory is used for safely approximating the $s$-semantics of definite logic programs. By taking equality theories which enforce so called non-terms elements to appear in the domain, one can obtain approximate $s$-models which encode non declarative properties of computed answer substitutions such as freeness. It is possible to formulate equality theories which lead to several well known abstract domains.
\end{abstract}

\section{Introduction}

The paper develops a new technique for abstract interpretation of definite logic programs which approximates the so-called $s$-semantics $[14,7]$. By including non ground atoms in the $s$-model, $s$-semantics describes not only declarative semantics, but also the computed answer substitutions for queries. The atoms in the $s$-model are representatives of equivalence classes of atoms where the equivalence relation is a variance (renaming) relation. The $s$-model can be computed by the non-ground $\mathbf{T}_{P}^{s}$-operator. The crucial step in applying this operator is the unification operation. Unification is characterised by the so-called Free Equality Theory (or Clarks Equality).

Our basic idea is to replace the Free Equality Theory by different weaker equality theories, which safely approximate it. Having selected a weaker theory, one can use model generation techniques $[6,12]$ to generate an interpretation of the equality predicate. This involves selecting a (typically finite) domain and determining the interpretation of all functors. The obtained interpretation safely approximates standard unification.

Applying the $\mathbf{T}_{P}^{s}$-operator using the approximating interpretation instead of standard unification yields an approximation of the $s$-model. If the domain of

\footnotetext{
* Supported by the Katholieke Universiteit Leuven. Permanent address: Keldysh Institute for Applied Mathematics, Russian Academy of Science, Miusskaya sq., 4, 125047 Moscow, Russia.

** Supported by the Belgian National Fund for Scientific Research.

** Supported by the Katholieke Universiteit Leuven Research Coordination.
} 
the approximating interpretation is finite, then the approximate $s$-model is finite and is obtained after a finite number of steps.

Safely approximating the $s$-model involves a subtlety. We use equality theories which enforce the existence of so-called non-term elements, i.e. elements which are not an interpretation of any Herbrand term. Their existence allows to infer freeness and dependency information in computed answers of most general queries. Of course, by applying magic set transformation [1], one can obtain information about call patterns arising in the execution of any query [8].

The level of detail obtained in the result of the analysis is determined by the chosen equality theory, especially the number of non-term elements is important. The notion of precision plays an important role in any abstract interpretation framework. In our setting it receives a rather simple and flexible meaning: it is determined by the set of equivalence classes of tuples of terms. Equivalent tuples of terms are not distinguishable, and, therefore, the results of the static analysis are precisely the properties which are distinguishable up to the equivalence relation. This treatment of precision allows to "implement" it as a model of a first order theory. In this respect our view is related to work in [15], where types are described by a definite logic program and used to abstract ground bottom-up computations. We generalise this, we use a model of a first order theory to describe equivalence classes (types), and use them to abstract non-ground bottom up computations.

Section 2 introduces background material, in particular on equality theories with non-term elements. Section 3 introduces interpretations containing nonterm elements. Section 4 develops the least fixpoint computation. Section 5 discusses the correctness and flexibility of the framework and sketches the method for deriving properties of a program. Section 6 summarises and discusses our results.

\section{Preliminaries: A First Order Language}

\subsection{Standard Notions}

Our formalisation of first order languages mostly follows Lloyd [20]. A language $\mathcal{L}$ is based on a so called signature $\Sigma=\langle F, P\rangle$, where $F \neq \emptyset$ ( $F$ are function symbols), $P \neq \emptyset$ ( $P$ are predicate symbols), $F \cap P=\emptyset$, and on a countable set of variables $\mathcal{V}$. $\operatorname{Terms}(F, \mathcal{V})$ denotes the set of terms constructed from $F$ and $\mathcal{V}$.

Given a well-formed formula $F$ based on $\mathcal{L}, \forall \mathcal{V}: F$ and $\exists \mathcal{V}: F$ denotes universal and existential closure of $F$, respectively. Also, given variables $X_{1}, \ldots, X_{n} \in \mathcal{V}$,

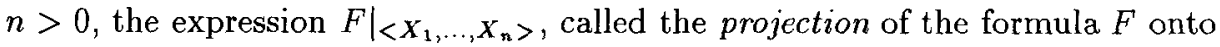
the tuple of variables $\left\langle X_{1}, \ldots, X_{n}\right\rangle$, is a shorthand for $\exists \mathcal{V}-\left\{X_{1}, \ldots, X_{n}\right\}: F$. Given a syntactical object $O$ based on $\mathcal{L}$, var $(O)$ denotes the set of free variables occurring in $O$. Syntactical objects $O$ and $O^{\prime}$ are said to be independent, noted $O$ ind $O^{\prime}$, iff $\operatorname{var}(O) \cap \operatorname{var}\left(O^{\prime}\right)=\emptyset$. We denote by $\bar{O}$ a tuple of pairwise distinct syntactical objects. In particular, $\bar{X}$ always denotes the tuple of distinct variables $<X_{1}, \ldots, X_{n}>, n>0$. Given a syntactical object $O$ of $\mathcal{L}, O \cong$ denotes an

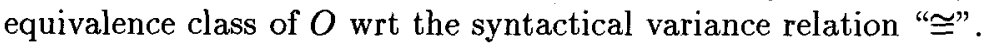


An interpretation I for a first order language is a triple $\langle\mathrm{D}, \Lambda, \mathcal{H}\rangle$, where $\mathrm{D}$ is the domain of the interpretation (a non-empty set), $\Lambda$ is an assignment of mappings of the form $D \times D \times \cdots \times D \mapsto D$ to the function symbols of $F$ and $\mathcal{H}$ is a truth function which maps atoms $p\left(\partial_{1}, \ldots, \partial_{n}\right)$ with $\partial_{1}, \ldots, \partial_{n} \in \mathbf{D}, n \geq 0$, $p^{(n)} \in \mathrm{P}$ to a truth value from $\{f, t\}$ where $\mathbf{f}<t, f^{-1}=t$ and $t^{-1}=f$. The truth function is determined by the set of atoms mapped to $t$. Given an interpretation $\mathbf{I}=\langle\mathrm{D}, A, \mathcal{H}\rangle$, the pair $\langle\mathrm{D}, \Lambda\rangle$, noted $\mathbf{I}_{\mathbf{o}}$, is called a pre-interpretation. A Herbrand interpretation of $\mathcal{L}$ has the Herbrand universe, noted $\mathrm{HU}_{\mathcal{L}}$, as domain.

Given an interpretation $\mathbf{I}=\langle\mathrm{D}, \Lambda, \mathcal{H}\rangle$ of $\mathcal{L}$ with signature $\Sigma=\langle\mathrm{F}, \mathrm{P}\rangle$, we denote by:

- $f$ and $p, \mathrm{t}, \mathrm{s}$ and $\overline{\mathrm{t}}, \overline{\mathrm{s}}$ function and predicate symbols, terms and tuples of terms based on $\mathcal{L}$, respectively

- $\partial$ and $\bar{\partial}$ a domain element of $D$ and a tuple of domain elements of $\bar{D}$, respectively

- I $(p), p^{(n)} \in \mathbf{P}, n \geq 0$ the set $\{p(\bar{\partial}) \mid \bar{\partial} \in \overline{\mathrm{D}}, \mathcal{H}(p(\bar{\partial}))=\mathbf{t}\}$

- I $(f)(\bar{\partial}), f \in \mathrm{F}, \bar{\partial} \in \overline{\mathrm{D}}$ a domain element $\hat{\partial} \in \mathrm{D}$ such that $f(\bar{\partial}) \mapsto \hat{\partial} \in \Lambda$

Given two interpretations $\mathbf{I}_{1}=\left\langle\mathrm{D}_{1}, \Lambda_{1}, \mathcal{H}_{1}>\right.$ and $\mathbf{I}_{2}=<\mathrm{D}_{2}, \Lambda_{2}, \mathcal{H}_{2}>$ of $\mathcal{L}$, a homomorphism $\kappa$ from $\mathbf{I}_{1}$ onto $\mathbf{I}_{2}$, noted $\mathbf{I}_{1} \stackrel{\kappa}{\rightarrow} \mathbf{I}_{2}$, is a mapping $\kappa: D_{1} \rightarrow D_{2}$ which satisfies the following conditions:

$-\kappa\left(D_{1}\right)=D_{2}$, i.e. $\kappa$ is a mapping "onto"

$-\kappa\left(\mathbf{I}_{1}(f)(\bar{\partial})\right)=\mathbf{I}_{2}(f) \kappa(\bar{\partial})$ for any function symbol $f$ of $\mathcal{L}$ and any $\bar{\partial} \in \overline{\mathrm{D}}_{1}$

$-\mathbf{I}_{1} \vDash p(\bar{\partial}), \bar{\partial} \in \bar{D}_{1}$ implies $\mathbf{I}_{2} \vDash p(\kappa(\bar{\partial}))$ for any predicate symbol $p$ of $\mathcal{L}$

Finally, an isomorphism $\lambda$ between interpretations $\mathbf{I}_{1}$ and $\mathbf{I}_{2}$, noted $\mathbf{I}_{1} \stackrel{\lambda}{\leftrightarrow} \mathbf{I}_{2}$, is a one-to-one correspondence (bijection) between the domains of the interpretations which preserves functions and predicates.

\subsection{Domain Terms and Domain Formulas}

We borrow some notations from [13]. Given a first order language $\mathcal{L}$ having signature $\Sigma=<\mathrm{F}, \mathrm{P}>$ and its interpretation $\mathrm{I}$ with domain $\mathrm{D}$, we extend the notion of syntactic object based on $\mathcal{L}$ (see section 2.1 ) by allowing domain elements $\partial$ of $\mathrm{D}$ to appear in them. Domain terms / atoms / formulas are terms/atoms/formulas containing elements of D. Ground domain atoms based on $\mathcal{L}$ and $I$ are called domain facts.

The interpretation $\mathbf{I}$ induces a unique mapping $\tilde{\mathbf{I}}$ on closed domain terms and domain facts which is defined inductively as follows: for any domain element $\partial$ : $\tilde{\mathbf{I}}(\partial)=\partial$ and

- for any $f^{(n)} \in \mathrm{F}, n \geq 0: \tilde{\mathbf{I}}\left(f\left(\mathrm{t}_{1}, \ldots, \mathrm{t}_{n}\right)\right)=\mathbf{I}(f)\left(\tilde{\mathbf{I}}\left(\mathrm{t}_{1}\right), \ldots, \tilde{\mathbf{I}}\left(\mathrm{t}_{n}\right)\right)$

- for any $p^{(n)} \in \mathbf{P}, n \geq 0: \tilde{\mathbf{I}}\left(p\left(t_{1}, \ldots, t_{n}\right)\right)=p\left(\tilde{\mathbf{I}}\left(\mathrm{t}_{1}\right), \ldots, \tilde{\mathbf{I}}\left(t_{n}\right)\right)$

where $t_{1}, \ldots, t_{n}$ are closed domain terms based on $\mathcal{L}$ and $I$.

A variable assignment $\mathbf{V}$ is a set of tuples $X / \mathrm{t}$ with $X$ a variable and $\mathrm{t}$ a domain term. Given a syntactical object $O, \mathrm{~V}(O)$ denotes the result of a 
variable assignment, i.e. $\mathbf{V}(O)$ is an instance of $O$. The truth function $\mathcal{H}_{\mathbf{I}}$ of the interpretation $\mathbf{I}$ can be extended to all closed domain formulas as follows: for any ground domain atom $p\left(\mathrm{t}_{1}, \ldots, \mathbf{t}_{n}\right): \mathcal{H}_{\mathbf{I}}\left(p\left(\mathrm{t}_{1}, \ldots, \mathrm{t}_{n}\right)\right)=\mathcal{H}_{\mathbf{I}}\left(p\left(\tilde{\mathbf{I}}\left(\mathrm{t}_{1}\right), \ldots, \tilde{\mathbf{I}}\left(\mathrm{t}_{n}\right)\right)\right)$; for any closed domain formula $F: \mathcal{H}_{\mathbf{I}}(\neg F)=\mathcal{H}_{\mathbf{I}}(F)^{-1}$ and

$$
\begin{aligned}
& \mathcal{H}_{\mathbf{I}}(F \vee G)=\max \left\{\mathcal{H}_{\mathbf{I}}(F), \mathcal{H}_{\mathbf{I}}(G)\right\} \quad \mathcal{H}_{\mathbf{I}}(F \wedge G)=\min \left\{\mathcal{H}_{\mathbf{I}}(F), \mathcal{H}_{\mathbf{I}}(G)\right\} \\
& \mathcal{H}_{\mathbf{I}}(F \leftrightarrow G)=\mathcal{H}_{\mathbf{I}}(F \wedge F \vee \neg F \wedge \neg G) \quad \mathcal{H}_{\mathbf{I}}(F \leftarrow G)=\mathcal{H}_{\mathbf{I}}(F \vee \neg G) \\
& \mathcal{H}_{\mathbf{I}}\left(\exists_{X}: F\right)=\max \left\{\mathcal{H}_{\mathbf{I}}(\{X / \partial\}(F)) \mid \partial \in \mathrm{D}\right\} \\
& \mathcal{H}_{\mathbf{I}}\left(\forall_{X}: F\right)=\min \left\{\mathcal{H}_{\mathbf{I}}(\{X / \partial\}(F)) \mid \partial \in \mathrm{D}\right\}
\end{aligned}
$$

This extension of the truth function enables us to consider models of closed domain formulas. Given an interpretation $\mathbf{I}$ and a closed (domain) formula $F$, we write $\mathbf{I} \vDash F$ iff $\mathcal{H}_{\mathbf{I}}(F)=\mathbf{t}$. This extends to $\mathbf{I} \vDash \mathcal{T}, \mathcal{T}$ being a theory in the classical way. A model of $\mathcal{T}$ is denoted by $\mathbf{M}_{\mathcal{T}}$. Notice that the interpretation of formulas without domain elements is unchanged.

\subsection{Equality Theories}

Let $\mathcal{L}$ be a first order language with equality, i.e. the set of predicate symbols $\mathrm{P}$ includes the equality predicate $=^{(2)}$. An equality theory based on $\mathcal{L}$ is a first order theory based on $\mathcal{L}$ such that the only predicate symbol occurring in it is the equality predicate. Given a pair of tuples of terms $\overline{\mathrm{t}}$ and $\overline{\mathrm{s}}$ having the forms $t_{1}, \ldots, t_{n}$ and $s_{1}, \ldots, s_{n}, n>0$, respectively, we denote by $\bar{t}=\vec{s}$ the equality formula $t_{1}=s_{1} \wedge \cdots \wedge t_{n}=s_{n}$.

The most weak equality theory is the standard Equality theory of $\mathcal{L}$, noted $E Q(\mathcal{L})$

$\begin{array}{ll}\text { Equivalence Axioms } & \text { Congruence Axioms } \\ \forall_{\mathcal{V}}: X=X & \forall_{\nu}: p(\bar{X}) \leftarrow p(\bar{Y}), \bar{X}=\bar{Y} \\ \forall_{\mathcal{V}}: X=Y \leftarrow Y=X & p^{(m)} \in \mathrm{P}, m>0 \\ \forall_{\mathcal{v}}: X=Z \leftarrow X=Y \wedge Y=Z & \end{array}$

Given an interpretation I of $\mathcal{L}$ with domain D satisfying the axioms of $E Q(\mathcal{L})$ (I is a model of $E Q(\mathcal{L})$ ), the equality predicate is an equivalence relation over elements of the domain $\mathrm{D}$. This equivalence relation forms a congruence for all predicates and functions of $\mathcal{L}$. Interpreting $=^{(2)}$ as identity, i.e. $\mathbf{I}(=)=$ $\{\partial=\partial \mid \partial \in \mathrm{D}\}$, one obtains a model of $E Q(\mathcal{L})$. This is done in the sequel.

The second important equality theory is the Free Equality theory (or Clarks Equality Theory), noted $F E Q(\mathcal{L})$. It extends $E Q(\mathcal{L})$ with:

$$
\begin{array}{ll}
\forall \mathcal{V}: f(\bar{X})=f(\bar{Y}) \rightarrow \bar{X}=\bar{Y} & \text { for any } f^{(n)} \in \mathrm{F}, n>0 \\
\forall \mathcal{V}: \neg(g(\bar{X})=f(\bar{Y})) & \text { for any } f, g \in \mathrm{F} \text { such that } f \neq g \\
\forall \mathcal{V}: \neg(\mathrm{t}=X) & \text { for any } \mathrm{t} \text { of } \mathcal{L} \text { containing the variable } X
\end{array}
$$

These additional axioms are not definite clauses (the axioms of $E Q(\mathcal{L})$ are). The Free Equality axioms formalise unification [20]; it is well-known (e.g., [13]) that $F E Q(\mathcal{L})$ implies the unification algorithm of Martelli and Montanari [21, 19]. A 
Herbrand interpretation satisfies $F E Q$-axioms if the equality predicate " $={ }^{(2)}$ " is interpreted as syntactical identity over the Herbrand universe.

The third equality theory is not so well-known, it is the Non-Term Equality Theory with at least $k$ non-term elements (see, for example, $[22]^{4}$ ), noted $N T E_{k}(\mathcal{L}), k>0$. It extends $E Q(\mathcal{L})$ with:

$$
\exists_{X_{1}, \cdots, X_{k}}\left(\bigwedge_{j=1}^{k} \operatorname{NTE}\left(X_{j}\right) \underset{\{i \neq j \mid i, j=1, \ldots, k\}}{\wedge} \neg\left(X_{i}=X_{j}\right)\right)
$$

where $\operatorname{NTE}(X)$ denotes the formula $\forall_{\bar{Y}_{1}, \ldots, \bar{Y}_{n}} \bigwedge_{i=1}^{n} \neg\left(X=f_{i}\left(\bar{Y}_{i}\right)\right)$ such that $f_{i} \in \mathrm{F}, i=1, \ldots, n$ are all function symbols of $\mathcal{L}$ and $\bar{Y}_{i}$ are the corresponding tuples of pairwise distinct variables. Notice that $\operatorname{NTE}(X)$ is not an atom.

The Non-Term Equality theory $N T E_{k}, k>0$ states that there exist at least $k$ objects which are not terms, i.e. domain elements which are not in the range of any function (in section 3 this property is exploited to represent variables). Therefore, $N T E_{k}(\mathcal{L}), k>0$ has no Herbrand model.

Following [22], the equality theories $E Q, F E Q, N T E_{k}$ and $N T E_{\infty}$ (the latter denotes the set $\left.\left\{N T E_{1}, N T E_{2}, \ldots\right\}\right)$ are used as a convenient toolkit to characterise pre-interpretations.

\section{3 nte-Interpretations: An Abstract Domain Construction}

Let $\mathcal{L}$ be a first order equality language with signature $\Sigma=\langle\mathrm{F}, \mathrm{P}\rangle$, where $\mathrm{F}$ is a non empty set of function symbols and $\mathbf{P}=\left\{=^{(2)}\right\}$. Suppose that $I$ is an interpretation of $\mathcal{L}$ with domain $\mathrm{D}$ such that $\mathbf{I} \models N T E_{k}(\mathcal{L}), k>0$, i.e. the domain $\mathrm{D}$ of $\mathbf{I}$ contains at least $k$ non-term elements. The latter allows to extend the mapping $\tilde{\mathbf{I}}$ as follows: given a variable $X \in \mathcal{V}$, we define $\tilde{\mathbf{I}}(X)=v$, where $v \in \mathrm{D}$ is one of the non-term domain elements. We assume that given an interpretation with non-term domain elements, the mapping of variables $\mathcal{V}$ to the non-term elements is a mapping "onto", which is fixed. For instance, with $\mathcal{V}=\left\{X_{0}, X_{1}, X_{2}, \ldots\right\}, X_{i}$ is mapped to $v_{i \bmod k}$ with $k$ the number of non-term elements.

\section{Definition 1. nte-Interpretation}

An interpretation $\mathbf{I}=\langle\mathrm{D}, \Lambda, \mathcal{H}\rangle$ of an equality language $\mathcal{L}$ is called a nteinterpretation provided that $\mathbf{I} \vDash N T E_{k}(\mathcal{L}), k>0$ and that $\partial \in \mathrm{D}$ iff there exists a term $\mathrm{t}$ of $\mathcal{L}$ such that $\tilde{\mathbf{I}}(\mathbf{t})=\partial$.

The above definition introduces two important conditions. The first one is a logical condition, which states that any nte-interpretation is a model of a nonterm equality theory. The second condition is a very strict non-logical domain closure axiom, which allows only domain elements which are the image under $\tilde{\mathbf{I}}$ of some term $t$.

\footnotetext{
${ }^{4}$ In [22] the non-term domain elements are called "roots" or isolated elements.
} 
Given a nte-interpretation $\mathbf{I}$, def. 1 enables us to define the mapping $\tilde{\mathbf{T}}: \mathrm{D} \rightarrow$ $\wp$ Terms $(\mathcal{L})$, which is the reverse of the mapping $\tilde{\mathbf{I}}$, as follows: for any domain element $\partial \in D: \tilde{\mathbf{T}}(\partial)=\{t \in \operatorname{Terms}(\mathcal{L}) \mid \tilde{\mathbf{I}}(\mathrm{t})=\partial\}$. We extend the mappings $\tilde{\mathbf{I}}$ and $\tilde{\mathbf{T}}$ to tuples and sets in the obvious way, e.g. given a tuple of terms $\overline{\mathbf{t}}=\left\langle\mathrm{t}_{1}, \ldots, \mathrm{t}_{n}>, n>0\right.$, we have that $\tilde{\mathbf{I}}(\overline{\mathrm{t}})=<\tilde{\mathbf{I}}\left(\mathrm{t}_{1}\right), \ldots, \tilde{\mathbf{I}}\left(\mathrm{t}_{n}\right)>$.

Example 1. Consider a language $\mathcal{L}$ with $F=\left\{[]^{(0)}, a^{(0)}, b^{(0)},[-H]^{(2)}\right\}$ and $P=$ $\left\{=^{(2)}\right\}$. Let $\mathbf{I}_{\mathbf{O}}=\langle\mathrm{D}, \Lambda\rangle$ be the following pre-interpretation of $\mathcal{L}$ :

- the domain D of the interpretation is the set $\left\{v, \gamma_{c}, \gamma_{f}, \iota\right\}$

- the mapping $\Lambda$ of the function symbols of $F$ :

$$
\begin{array}{llllll}
{[] \mapsto \gamma_{c}} & {\left[\gamma_{c} \mid \gamma_{c}\right] \mapsto \gamma_{f}} & {\left[\gamma_{f} \mid \gamma_{c}\right] \mapsto \gamma_{f}} & {[v \mid *] \mapsto \iota} & {[\iota \mid *] \mapsto \iota} \\
a \mapsto \gamma_{c} & {\left[\gamma_{c} \mid \gamma_{f}\right] \mapsto \gamma_{f}} & {\left[\gamma_{f} \mid \gamma_{f}\right] \mapsto \gamma_{f}} & {[* \mid v] \mapsto \iota} & {[* \mid \iota] \mapsto \iota} \\
b \mapsto \gamma_{c} & & &
\end{array}
$$

where " $*$ " denotes any element of $D$. Notice that $v$ is the only non-term element, i.e. $\tilde{\mathbf{I}}(X)=v$ for all variables $X$. Also, observe that if $\mathbf{I}=\mathbf{I}_{\mathbf{0}} \cup \mathbf{I}(=)$, where "=" is identity over $\mathrm{D}$, then $\mathbf{I}=N T E_{1}(\mathcal{L})$. We have that $\tilde{\mathbf{T}}\left(\gamma_{c}\right)=\{[, a, b\}$, i.e. it is the set of constants, $\mathbf{T}\left(\gamma_{f}\right)$ is the set of all ground terms, which are not constants, $\tilde{\mathbf{T}}(v)=\mathcal{V}$, and $\tilde{\mathbf{T}}(\iota)$ is the set of all remaining terms (instantiated terms).

A nte-interpretation of $\mathcal{L}$ is called minimal, denoted $\mathbf{I}^{\mathrm{min}}$, if its domain contains the minimal number of elements. The domain $D^{\text {min }}$ of the minimal nteinterpretation $\mathbf{I}^{\mathrm{min}}$ of $\mathcal{L}$ consists of two domain elements, e.g. $v$ and $\partial$, where $v$ is a non-term element and $\partial$ is the image of any non-variable term $t$ under $\tilde{\mathbf{I}}$ Notice that all minimal nte-interpretations of $\mathcal{L}$ are isomorphic and that for any minimal interpretation $\mathbf{I}^{\text {min }}$ of $\mathcal{L}$ holds: $\mathbf{I}^{\text {min }} \models N T E_{1}$, i.e. it is a model of the most weak non-term equality theory.

An $n t e$-interpretation is called maximal, denoted $\mathbf{I}^{\max }$, if for any pair of different domain elements $\partial, \partial^{\prime}$ holds: $\tilde{\mathbf{I}}(\mathrm{t})=\partial$ and $\tilde{\mathbf{I}}\left(\mathrm{t}^{\prime}\right)=\partial^{\prime}$ iff $\mathrm{t}$ is syntactically different from $t^{\prime}$. The domain of a maximal nte-interpretation contains as many domain elements as there are terms in $\operatorname{Terms}(F, \mathcal{V})$. All maximal $n t e$-interpretations are isomorphic, because for maximal nte-interpretations the mapping $\tilde{\mathbf{I}}^{\max }$ is an isomorphism between the set of all terms of $\mathcal{L}$ and the domain $D^{\max }$ of $\mathbf{I}^{\max }$, which preserves all functions of $\mathcal{L}$. Therefore, $\mathbf{I}^{\max } \vDash$ $F E Q(\mathcal{L}) \cup N T E_{\infty}(\mathcal{L})$, i.e. it is a model of the most precise non-term equality theory.

Given an equality language $\mathcal{L}, \mathcal{I}^{\text {nte }}(\mathcal{L})$ denotes the class of all nte-interpretations of $\mathcal{L}$. In the sequel we distinguish nte-interpretations of $\mathcal{L}$ modulo isomorphism. This implies that the set $\mathcal{I}^{n t e}(\mathcal{L})$ contains exactly one minimal interpretation and exactly one maximal interpretation. Recall that the equality predicate is always interpreted as identity. Therefore, given an equality language $\mathcal{L}$, we can only consider the set of all pre-interpretations $\mathcal{I}_{\mathbf{0}}{ }^{\text {nte }}(\mathcal{L})$ of the corresponding nte-interpretations. 
Let $\mathbf{I}_{\mathbf{0}}{ }^{\prime}=<D^{\prime}, \Lambda^{\prime}>$ and $\mathbf{I}_{\mathbf{0}}{ }^{\prime \prime}=<D^{\prime \prime}, \Lambda^{\prime \prime}>$ be a pair of pre-interpretations of $\mathcal{I}_{\mathbf{o}}{ }^{\text {nte }}(\mathcal{L})$. Without loss of generality we can assume that $D^{\prime} \cap D^{\prime \prime}=\emptyset$. The composition $\mathbf{I}_{\mathbf{o}}{ }^{\prime} \odot \mathbf{I}_{\mathbf{o}}{ }^{\prime \prime}$ of pre-interpretations is a pre-interpretation $\mathbf{I}_{\mathbf{o}}=\langle\mathrm{D}, \Lambda\rangle$ of $\mathcal{L}$, which is defined as follows. Consider the set $\hat{D}=D^{\prime} \cup D^{\prime \prime}$. Let " $\sim$ " be the minimal equivalence relation over $\hat{D}$ such that for any $\partial^{\prime} \in D^{\prime}$ and $\partial^{\prime \prime} \in D^{\prime \prime}$ : $\tilde{\mathbf{T}}^{\prime}\left(\partial^{\prime}\right) \cap \tilde{\mathbf{T}}^{\prime \prime}\left(\partial^{\prime \prime}\right) \neq \emptyset$ implies $\partial^{\prime} \sim \partial^{\prime \prime}$.

The domain of $I_{0}$ is defined as $D=\hat{D}^{\sim}$, i.e. the domain of $I_{0}$ is a set of equivalence classes induced by the equivalence relation " $\sim$ " over the elements of $\hat{D}$. It is important to notice that the domain closure axiom of def. 1 ensures that for any $\partial^{\prime} \in \mathrm{D}^{\prime}$ there exists $\partial^{\prime \prime} \in \mathrm{D}^{\prime \prime}$ such that $\partial^{\prime} \sim \partial^{\prime \prime}$ and vice versa. Let $\partial^{\sim}$ denote an equivalence class of $\partial \in \hat{\mathbf{D}}$, then the corresponding mapping $\Lambda$ satisfies: for any $f^{(n)} \in \mathrm{F}, n \geq 0$ holds: $f\left(\partial_{1} \sim, \ldots, \partial_{n} \sim\right) \mapsto \partial^{\sim} \in \Lambda$ iff $f\left(\partial_{1}, \ldots, \partial_{n}\right) \mapsto \hat{\partial} \in \Lambda^{\prime} \cup \Lambda^{\prime \prime}$ Obviously $\mathbf{I}_{\mathbf{O}}$ is a pre-interpretation of $\mathcal{L}$ (the composition is defined correctly). Therefore, the set $\mathcal{I}_{\mathbf{o}}{ }^{\text {nte }}(\mathcal{L})$ is closed wrt composition of pre-interpretations. Moreover, $\mathbf{I}$ is an nte-interpretation of $\mathcal{L}$ if $\mathbf{I}(=)$ is identity over $D$.

Indeed, the above discussion implies that given $\mathbf{I}_{\mathbf{0}}=\mathbf{I}_{\mathbf{0}}{ }^{\prime} \odot \mathbf{I}_{\mathbf{0}}{ }^{\prime \prime}$, there exists a pair of homomorphisms $\mathbf{I}_{\mathbf{o}}{ }^{\prime} \stackrel{\kappa^{\prime}}{\rightarrow} \mathbf{I}_{\mathbf{O}}$ and $\mathbf{I}_{\mathbf{0}}{ }^{\prime \prime} \stackrel{\kappa^{\prime \prime}}{\rightarrow} \mathbf{I}_{\mathbf{0}}$. The homomorphism $\boldsymbol{\kappa}^{\prime}$ is the following mapping: for any $\partial^{\prime} \in \mathrm{D}^{\prime}$ holds: $\kappa^{\prime}\left(\partial^{\prime}\right)=\partial$, where $\partial \in \mathrm{D}$ is the equivalence class of $\partial^{\prime}$ in $D$. The homomorphism $\kappa^{\prime \prime}$ is defined in a similar way.

The set $\mathcal{I}_{\mathbf{o}}{ }^{\text {nte }}(\mathcal{L})$ can be equipped with an order relation " $\preceq$ ": given $\mathbf{I}_{\mathbf{o}}{ }^{\prime}, \mathbf{I}_{\mathbf{o}}{ }^{\prime \prime} \in$ $\mathcal{I}_{\mathbf{o}}{ }^{\text {nte }}(\mathcal{L}), \mathbf{I}_{\mathbf{0}}{ }^{\prime \prime}$ is said to be more precise than $\mathbf{I}_{\mathbf{o}}{ }^{\prime}$, noted $\mathbf{I}_{\mathbf{0}}{ }^{\prime} \preceq \mathbf{I}_{\mathbf{o}}{ }^{\prime \prime}$, iff $\mathbf{I}_{\mathbf{o}}{ }^{\prime}=$ $\mathbf{I}_{\mathbf{o}^{\prime}} \odot \mathbf{I}_{\mathbf{o}}{ }^{\prime \prime}$. It is clear that for any $\mathbf{I}_{\mathbf{o}} \in \mathcal{I}_{\mathbf{o}}{ }^{\text {nte }}(\mathcal{L}): \mathbf{I}_{\mathbf{o}}{ }^{\min } \preceq \mathbf{I}_{\mathbf{o}} \preceq \mathbf{I}_{\mathbf{o}}{ }^{\text {max }}$. Notice that $\mathbf{I}_{\mathbf{o}}{ }^{\prime} \preceq \mathbf{I}_{\mathbf{o}}{ }^{\prime \prime}$ iff there exists a homomorphism $\mathbf{I}_{\mathbf{o}}{ }^{\prime \prime} \stackrel{\kappa}{\rightarrow} \mathbf{I}_{\mathbf{o}}{ }^{\prime}$. Also, $\mathbf{I}_{\mathbf{o}}{ }^{\prime} \preceq \mathbf{I}_{\mathbf{o}}{ }^{\prime \prime}$ and $\mathbf{I}_{\mathbf{0}}{ }^{\prime \prime} \preceq \mathbf{I}_{\mathbf{o}}{ }^{\prime}$ iff $\mathbf{I}_{\mathbf{o}}{ }^{\prime}$ and $\mathbf{I}_{\mathbf{o}}{ }^{\prime \prime}$ are isomorphic.

Definition 2. Equivalence of tuples of terms modulo nte-Interpretation Given an equality language $\mathcal{L}$ and a nte-interpretation $I$ of $\mathcal{L}$, two tuples of terms $\overline{\mathbf{t}}$ and $\overline{\mathbf{s}}$ of $\operatorname{Terms}(\mathcal{L})$ having the same arity are said to be variants of each other modulo nte-interpretation $\mathbf{I}$, denoted $\overline{\mathrm{t}} \approx_{\mathbf{I}} \overline{\mathbf{s}}$, iff

$$
\mathbf{I} \vDash \forall_{\bar{X}}:\left.\left.(\bar{X}=\overline{\mathrm{t}})\right|_{\bar{X}} \leftrightarrow(\bar{X}=\overline{\mathrm{s}})\right|_{\bar{X}}
$$

where $\bar{X}$ ind $\{\overline{\mathbf{t}}, \overline{\mathbf{s}}\}$.

Proposition 3. Given an equality language $\mathcal{L}$; for any pair of tuples of terms $\overline{\mathrm{t}}$ and $\overline{\mathbf{s}}$ having the same arity holds:

$-\overline{\mathrm{t}} \cong \overline{\mathbf{s}}$ iff $\overline{\mathrm{t}} \approx_{\operatorname{Imax}} \overline{\mathbf{s}}$, where $\mathbf{I}^{\max }$ is the maximal nte-interpretation of $\mathcal{L}$

$-\overline{\mathbf{t}} \cong \overline{\mathbf{s}}$ implies $\overline{\mathrm{t}} \approx \overline{\mathbf{s}} \overline{\text { for }}$ fory nte-interpretation $\mathbf{I}$ of $\mathcal{L}$.

Any nte-interpretation $\mathbf{I}$ of $\mathcal{L}$ induces an equivalence relation over the set of tuples of terms of $\mathcal{L}$. Let $\Upsilon_{\mathbf{I}}(\mathcal{L})$ denote the set $\left\{\tau_{1}, \ldots, \tau_{n}, \cdots\right\}$ of equivalence classes (types) of terms of $\mathcal{L}$ wrt " $\approx \mathbf{I}$ " induced by $\mathbf{I}$. For instance, the nteinterpretation $I$ of example 1 induces the set of equivalence classes $r_{\mathbf{I}}(\mathcal{L})=$ 
$\left\{\tau_{v}, \tau_{\gamma_{c}}, \tau_{\gamma_{f}}, \tau_{l}\right\}$, where $\tau_{v}$ is the equivalence class of all variables, $\tau_{\gamma_{c}}$ and $\tau_{\gamma_{f}}$ are the equivalence classes of constants and ground non-constant terms, respectively, and $\tau_{\iota}$ is the equivalence class of all remaining terms (containing variables). Due to proposition 3 we have that $\Upsilon_{I \max }(\mathcal{L})=\{\mathrm{t} \cong \mid \mathrm{t}$ is a term of $\mathcal{L}\}$.

Given $k>0$, the set of equivalence classes of tuples of terms of $\mathcal{L}$ (types) of arity $k$ modulo $\mathbf{I}$ is noted $\bar{\Upsilon}_{\mathbf{I}}^{(k)}(\mathcal{L})$, which has the following properties:

- for any tuple $\overline{\mathrm{t}}$ of terms: there exists a type $\bar{\tau} \in \bar{\Upsilon}_{\mathbf{I}}$ such that $\overline{\mathrm{t}} \in \bar{\tau}$

- for any pair of tuples of terms $\overline{\mathrm{t}}^{\prime}$ and $\overline{\mathrm{t}}^{\prime \prime}: \overline{\mathrm{t}}^{\prime} \cong \overline{\mathrm{t}}^{\prime \prime}$ and $\overline{\mathrm{t}}^{\prime} \in \bar{\tau}$ implies $\overline{\mathrm{t}}^{\prime \prime} \in \bar{\tau}$

- for any pair of primitive types $\bar{\tau}^{\prime}, \bar{\tau}^{\prime \prime} \in \bar{\Upsilon}_{\mathbf{I}}: \overline{\mathrm{t}} \in \bar{\tau}^{\prime}$ and $\overline{\mathrm{t}} \in \bar{\tau}^{\prime \prime}$ implies $\bar{\tau}^{\prime}=\bar{\tau}^{\prime \prime}$

In other words, the set $\bar{\Upsilon}_{\mathbf{I}}(\mathcal{L})$ can be considered as a set of abstract tuples of terms, or types. The precision of the analysis is determined by the granularity of the equivalence classes which in turn is determined by the chosen equality theory. The corresponding nte-interpretation $\mathbf{I}$ of $\mathcal{L}$ can be produced by applying a model generation technique, e.g. the algorithms of $[6,12]$ are suitable. Equivalence classes can be specified by means of a special first order theory $\mathcal{T}$, called a precision theory. This gives rise to a significant generalisation of the approach of [18] and allows to use our approach for the generation of abstract domains for well-known abstract interpretations of definite logic programs. The precision theory $\mathcal{T}$ obtained as a formalisation of a particular problem (e.g. freeness and dependency analysis) forms an input for a model generator. For instance, the pre-interpretation of example 1 has been obtain as a pre-interpretation of the nte-model of the following precision theory $\mathcal{T}$ :

for each constant $f^{(0)} \in \mathrm{F}: \operatorname{ground}_{c}(f)$

for each function $f^{(k)} \in \mathrm{F}$ :

$$
\begin{aligned}
& \forall X_{1}, \ldots, X_{k} \operatorname{ground}_{f}\left(f\left(X_{1}, \ldots, X_{k}\right)\right) \leftarrow \bigwedge_{i=1}^{k}\left(\operatorname{ground}_{c}\left(X_{i}\right) \vee \operatorname{ground}_{f}\left(X_{i}\right)\right) \\
& \forall_{X_{1}, \ldots, X_{k}} \operatorname{inst}\left(f\left(X_{1}, \ldots, X_{k}\right)\right) \leftarrow \bigvee_{i=1}^{k}\left(\operatorname{inst}\left(X_{i}\right) \vee \operatorname{NTE}\left(X_{i}\right)\right)
\end{aligned}
$$

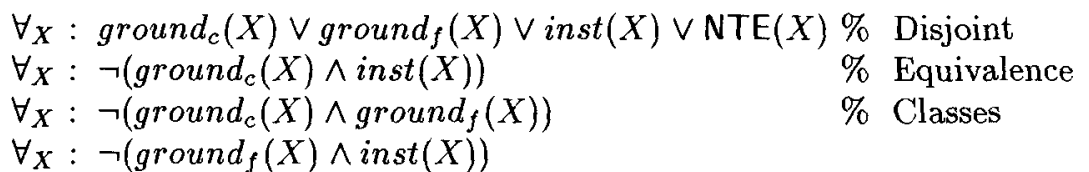

Notice that each equivalence class of the set $\left\{\tau_{\gamma_{c}}, \tau_{\gamma_{s}}, \tau_{\iota}\right\}$ is specified by the corresponding unary predicate of the set $\left\{\right.$ ground $_{c}^{(1)}$, ground $_{f}^{(1)}$, inst $\left.^{(1)}\right\}$. The equivalence class of all variables $\tau_{v}$ is automatically specified by $N T E_{1}$. This observation shows that our approach is related to the topics discussed in $[15,16]$.

In general, if there exists a finite model of the precision theory $\mathcal{T}$, then the finite nte-model $\mathbf{M}_{\mathcal{T}}^{\text {min }}$ with the minimal number of domain elements can be generated. In principle, the latter gives rise to an implementation technique for the automatic construction of abstract interpretation algorithms using $\bar{\Upsilon}_{\mathbf{I}}(\mathcal{L})$ as an abstract domain.

The remainder of the paper illustrates this idea by developing an abstraction of the s-semantics. The latter allows to obtain freeness and dependency analysis in a bottom-up manner. 


\section{Least Fixpoint Computations}

\subsection{Constraint Systems and Constraint Programs}

Let $\mathcal{L}$ be a first order equality language. The set $\mathcal{E}, \mathcal{E} \subseteq \mathcal{L}$, is called the set of constraints based on $\mathcal{L}$ iff it is the set of all well-formed formulas of $\mathcal{L}$ which are constructed only using equality atoms, connectives $\wedge$ and existential quantifiers $\exists$. We use a notion of constraint system which has been motivated by the generic approach suggested in $[11,17]-$ a constraint system based on $\mathcal{L}$, noted $\mathcal{S}$, is a pair $\langle\mathcal{E}, \mathbf{I}\rangle$, where $\mathbf{I}$ is a nte-interpretation of $\mathcal{L}$. A constraint $\mathbf{E} \in \mathcal{E}$ (from here on constraints will be given in bold) is said to be atomic, denoted $\mathbf{A}$, iff it is a non-empty conjunction of equality atoms. Also, a constraint is said to be in solved form, iff it has the form $\bar{X}=\overline{\mathrm{t}}, \bar{X}$ ind $\overline{\mathrm{t}}$, where $\overline{\mathrm{t}}$ is a tuple of terms of $\mathcal{L}$.

A constraint definite logic program based on $\mathcal{E}$, where $\mathcal{E}$ is the set of constraints based on $\mathcal{L}$, is denoted $P_{\mathcal{E}}$, and consists of finitely many constraint clauses having the form $\forall v:\left(p(\bar{X}) \leftarrow p_{1}\left(\bar{X}_{1}\right) \wedge \cdots \wedge p_{n}\left(\bar{X}_{n}\right)\right) \diamond \mathbf{A}, n \geq 0$, where $p, p_{1}, \cdots, p_{n}$ are program predicates not occurring in $\mathcal{L}$ and and $\bar{X}, \bar{X}_{1}, \cdots, \bar{X}_{n}$ are tuples of distinct variables; and $\mathbf{A}$ is an atomic constraint in $\mathcal{E}$.

A constraint atom based on $\mathcal{E}$ has the form $p(\bar{X}) \diamond \mathbf{E}, \mathbf{E} \in \mathcal{E}$, where $\bar{X} \subseteq$ $\operatorname{var}(\mathbf{E})$ and $p$ is a program predicate. Let $\mathcal{B}_{\mathcal{E}}$ denote the set of all constraint atoms based on $\mathcal{E}$. A constraint atom is in solved form iff it is of the form $p(\bar{X}) \diamond \bar{X}=\overline{\mathrm{t}}$ where $\bar{X}$ ind $\overline{\mathrm{t}}$.

\subsection{Bottom-up Fixpoint Constraint Computations}

Consider a constraint system $\mathcal{S}=\langle\mathcal{E}, \mathbf{I}\rangle$ based on $\mathcal{L}$, where $\mathbf{I}$ is a nteinterpretation of the equality language $\mathcal{L}$. Two constraint atoms $A, A^{\prime} \in \mathcal{B}_{\mathcal{E}}$ having the forms $p(\bar{X}) \diamond \mathbf{E}$ and $p\left(\bar{X}^{\prime}\right) \diamond \mathbf{E}^{\prime}$ respectively, are said to be variants of each other modulo $\mathbf{I}$, noted $A \approx_{\mathbf{I}} A^{\prime}$, iff

$$
\mathbf{I} \vDash \forall \nu:\left.\left.\left(\bar{Y}=\left.\bar{X} \wedge \mathbf{E}\right|_{\bar{X}}\right)\right|_{\bar{Y}} \leftrightarrow\left(\bar{Y}=\left.\bar{X}^{\prime} \wedge \mathbf{E}^{\prime}\right|_{\bar{X}^{\prime}}\right)\right|_{\bar{Y}}
$$

where $\bar{Y}$ ind $\left\{\bar{X}, \bar{X}^{\prime}\right\}$.

Given a constraint atom $A \in \mathcal{B}_{\mathcal{E}}$ and a set of constraint atoms Atoms $\in \wp\left(\mathcal{B}_{\mathcal{E}}\right)$, $A^{\approx I}$ and Atoms $\approx$ I denote an equivalence class of $A$ (quotient constraint atom) and a set of equivalence classes of atoms of Atoms (quotient set of constraint atoms), respectively.

Least fixpoint computations are performed over quotient sets of constraint atoms. Consider a constraint system $\mathcal{S}=\langle\mathcal{E}, \mathbf{I}\rangle$. Given a program $P_{\mathcal{E}}$, a quotient constraint immediate consequence operator modulo $\mathbf{I}, \mathbf{T}_{P_{\mathcal{E}}}^{\mathbf{I}}: \wp\left(\mathcal{B}_{\mathcal{E}}^{\approx} \mathbf{I}\right) \mapsto$ $\wp\left(\mathcal{B}_{\mathcal{E}}^{\approx} \mathbf{I}\right)$ can be defined as usual, e.g. in the style of $[20,14,17,7]$ : for any 
Atoms $\approx \mathbf{I} \in \wp\left(\mathcal{B}_{\mathcal{E}}^{\approx \mathbf{I}}\right)$ we define $\mathbf{T}_{P_{\mathcal{E}}}^{\mathbf{I}}($ Atoms $\approx \mathbf{I})=$

$$
\bigcup_{C l \in P_{\mathcal{S}}}\left\{\left[\left.p(\bar{X}) \diamond \hat{\mathbf{E}}\right|_{\bar{X}}\right]^{\approx \mathbf{I}} \mid \begin{array}{l}
C l: p(\bar{Y}) \leftarrow p_{1}\left(\bar{Y}_{1}\right) \wedge \cdots \wedge p_{n}\left(\bar{Y}_{n}\right) \diamond \mathbf{A} \\
\hat{\mathbf{E}}: \bar{X}=\bar{Y} \wedge \mathbf{E}_{1}^{\prime} \wedge \cdots \wedge \mathbf{E}_{n}^{\prime} \wedge \mathbf{A}, \bar{X} \text { ind } C l \\
\text { where for each } k=1,2, \cdots, n \text { holds }: \\
\mathbf{E}_{k}^{\prime}: \bar{X}_{k}=\bar{Y}_{k} \wedge\left(\left.\mathbf{E}_{k}\right|_{\bar{X}_{k}}\right) \text { is such that } \\
{\left[p_{k}\left(\bar{X}_{k}\right) \diamond \mathbf{E}_{k}\right] \mathbf{\sim} \mathbf{I} \in \text { Atoms } \approx \text { and }} \\
\bar{X}_{k} \text { ind }\{C l\} \cup\left\{\bar{X}, \bar{X}_{1}, \cdots, \bar{X}_{k-1}\right\}
\end{array}\right\}
$$

Given an interpretation $\mathbf{I}$ of $\mathcal{L}$, the partially ordered set $\left\langle\wp\left(\mathcal{B}_{\mathcal{E}}^{\approx} \mathbf{I}\right), \subseteq\right\rangle$ is a complete lattice and $\mathbf{T}_{P_{\mathcal{E}}}^{\mathbf{I}}$ is monotonic (wrt $\subseteq$ ) and continuous. Thus, as usual, the monotonicity and continuity imply that $\operatorname{Ifp}_{\mathbf{p}} \mathbf{T}_{P_{\varepsilon}}^{\mathbf{I}}=\mathbf{T}_{P_{\varepsilon}}^{\mathbf{I}} \uparrow \omega(\emptyset)$.

Given a constraint definite logic program $P_{\mathcal{E}}, \mathcal{O}\left(P_{\mathcal{E}}\right)$ denotes the $s$-semantics of $P_{\mathcal{E}}$. Syntactically, the $s$-semantics of a program is a quotient wrt $\cong$ set of atoms having the form $p\left(t_{1}, \cdots, t_{n}\right)$, where $t_{1}, \cdots, t_{n}$ are terms of $\mathcal{L}$ and $p$ is a program predicate (see for formal definition $[14,7]$ ). The proposition below shows an important relation between the least fixpoint computation and the $s$-semantics for definite logic programs:

Proposition 4. Given a constraint program $P_{\mathcal{E}}$, where $\mathcal{E}$ is based on $\mathcal{L}$.

$$
p(\overline{\mathrm{t}})=\mathcal{O}\left(P_{\mathcal{E}}\right) \text { iff }[p(\bar{X}) \diamond \bar{X}=\overline{\mathrm{t}}]^{\approx \mathbf{I}^{\max }} \in \operatorname{lfp} \mathbf{T}_{P_{\mathcal{E}}}^{\max }, \overline{\mathrm{t}} \text { ind } \bar{X}
$$

holds provided that $\mathbf{I}^{\max }$ is the maximal nte-interpretation of $\mathcal{L}$.

The importance of non-term elements can be illustrated by the following example. Consider the program

$$
p(X, Y) \leftarrow \diamond X=Y \quad p(X, Y) \leftarrow \diamond X=a \wedge Y=a
$$

If $\mathbf{I}$ is the standard intended Herbrand interpretation (i.e. no non-term elements), then the least fixpoint is $\left\{[p(X, Y) \diamond X=a \wedge Y=a]^{\approx} \mathbf{I}\right\}$, which is the least Herbrand model. If $\mathbf{I}$ is equipped with a non-term element, we obtain $\left\{[p(X, Y) \diamond X=Y]^{\approx} \mathbf{I},[p(X, Y) \diamond X=a \wedge Y=a]^{\approx \mathbf{I}}\right\}$, as least fixpoint, showing that there exist two different computed answer substitutions for the initial goal $p(X, Y)$.

Given a program $P_{\mathcal{E}}$, the least fixpoint computations can be controlled by taking different nte-interpretations of $\mathcal{I}^{\text {nte }}(\mathcal{L})$. These computations have the following property:

Proposition 5. Consider a constraint system $\mathcal{S}=\langle\mathcal{E}, \mathbf{I}\rangle$, where $\mathcal{E}$ is based on $\mathcal{L}$ and $\mathbf{I} \in \mathcal{I}^{\text {nte }}(\mathcal{L})$. Let $P_{\mathcal{E}}$ be a constraint definite logic program based on $\mathcal{E}$. Given an atom $A \in \mathcal{B}_{\mathcal{E}}$ such that $A^{\approx} \mathbf{I m a x} \in \mathbf{l} \mathbf{f} \mathbf{p} \mathbf{T}_{P_{\mathcal{E}}}^{\mathbf{I m a x}}$, there exists an atom $A^{\prime} \in \mathcal{B}_{\mathcal{E}}$ such that $A \approx \mathbf{I} \max A^{\prime}$ and $A^{\prime \approx} \mathbf{I} \in \mathbf{l} \mathbf{p} \mathbf{p} \mathbf{T}_{P_{\mathcal{E}}}^{\mathbf{I}}$. 
The equality constraints occurring in constraint atoms cannot be simplified using the standard notion of unification implied by the unification algorithm of Martelli and Montanari [21, 19]. This algorithm is only applicable if the preinterpretation satisfies the $F E Q$-axioms. However, a set of minimal simplification rules is applicable. It consists of all first order tautologies and the following rewriting rule: $\exists_{\{X\}}(X=\mathrm{t} \wedge \mathbf{E}) \Leftrightarrow\{X / \mathrm{t}\}(\mathbf{E})$, where $X$ ind $\mathrm{t}$. The correctness of this transformation is implied by the $E Q(\mathcal{L})$-axioms, and therefore by any nte-interpretation.

Example 2. Consider a constraint system $\mathcal{S}=\langle\mathcal{E}, \mathrm{I}\rangle$, where the equality language $\mathcal{L}$ and its nte-interpretation $I$ are defined as in example 1 . Then the program $A p p \mathcal{E}$ below

$$
\begin{aligned}
& \forall \mathcal{V}: p(L, Y) \leftarrow \operatorname{app}(X, L, Y) \diamond X=[a, b \mid Y] \\
& \forall \mathcal{V}: \operatorname{app}(X, L, Y) \diamond X=[] \wedge L=Y \\
& \forall \mathcal{V}: \operatorname{app}(X, L, Y) \leftarrow \operatorname{app}(T, L, R) \diamond X=[H \mid T] \wedge Y=[H \mid R]
\end{aligned}
$$

is a constraint definite logic program based on $\mathcal{E}$, where $p^{(2)}, a p p^{(3)}$ are program predicates. Then the least fixpoint $\mathbf{I f p}_{\mathbf{A p p \mathcal { E }}}^{\mathbf{I}}$ can be obtained as follows:

$\begin{array}{ll}\text { New Quotient Flat Constraint Atoms } & \text { Iteration } \\ {\left[\left.\operatorname{app}(X, L, Y) \diamond(X=[] \wedge L=Y)\right|_{<X, L, Y>}\right]_{\mathbf{I}}} & 1^{\text {st }} \\ {\left[\left.\operatorname{app}(X, L, Y) \diamond(X=[H] \wedge Y=[H \mid L])\right|_{<X, L, Y>}\right]_{\mathbf{I}} 2^{\text {nd }}} \\ {\left[\left.p(L, Y) \diamond([a, b \mid Y]=[H] \wedge Y=[H \mid L])\right|_{<L, Y>}\right]_{\mathbf{I}}} & 3^{\text {rd }} \\ \text { no more new elements } & 4^{\text {th }}\end{array}$

Notice that $[H]$ and $\left[H \mid H^{\prime}\right]$ are in the same equivalence class, so the third iteration does not produce a new app-atom. On the other hand []$=[a, b \mid Y]$ fails and $[a, b \mid Y]=[H]$ cannot be simplified by the "peel" operation of Martelli and Montanari $[21,19]$. Under $F E Q(\mathcal{L})$, one could apply "peel" and one would obtain that there are no $p$-atoms in the fixpoint. What can be extracted from the fixpoint will be discussed in the next section.

\section{An Abstract Interpretation Framework for s-semantics}

The constraint least fixpoint computation for $P_{\mathcal{E}}$ can be performed over $\mathcal{S}=<$ $\mathcal{E}, \mathbf{I}>$ using any nte-interpretation $\mathbf{I}$ of the equality language $\mathcal{L}$. Proposition 4 ensures that if $\mathbf{I}$ is maximal for $\mathcal{L}$, then the immediate consequence operator computes the $s$-semantics of $P_{\mathcal{E}}$, i.e. the intended (concrete) semantics of the given program. In our framework any nte-interpretation $\mathbf{I} \in \mathcal{I}^{\text {nte }}(\mathcal{L})$ is a safe abstract interpretation. It approximates the $s$-semantics of a given program with a precision, which is determined by the granularity of the equivalence classes of the set $\bar{\Upsilon}_{\mathbf{I}}(\mathcal{L})$ induced by $\mathbf{I}$ (see section 3 ).

Let $p(\overline{\mathrm{t}})$ be a program atom in the $s$-success set of $P_{\mathcal{E}}$, i.e. $p(\overline{\mathrm{t}})^{\simeq} \in \mathcal{O}\left(P_{\mathcal{E}}\right)$.

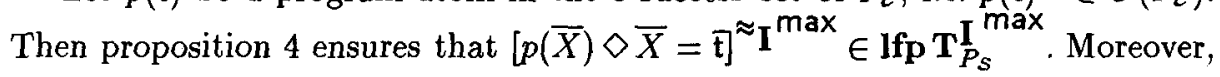


by proposition 5 we have that given a nte-interpretation $I \in \mathcal{I}^{\text {nte }}(\mathcal{L})$, there exists an atom $\left[\left.p(\bar{X}) \diamond \mathbf{E}\right|_{\bar{X}}\right]^{\approx} \mathbf{I} \in \mathbf{l f} \mathbf{p} \mathbf{T}_{P_{S}}^{\mathbf{I}}$ such that $\mathbf{I}^{\max } \vDash \forall_{\nu}:\left.\left.\mathbf{E}\right|_{\bar{X}} \leftrightarrow(\bar{X}=\overline{\mathbf{t}})\right|_{\bar{X}}$. Suppose that the constraint $\left.\mathbf{E}\right|_{\bar{X}}$ has, wrt $\mathbf{I}$, a solved form $\bar{X}=\overline{\mathbf{s}}, \tilde{\mathbf{s}}$ ind $\bar{X}$. It can be shown that the existence of a solved form and some assumptions concerning the structure of the least fixpoint $1 \mathbf{f} \mathbf{p} \mathbf{T}_{P_{S}}^{\mathbf{I}}$ (see discussion below) ensure that $\mathbf{I} \vDash \forall_{\mathcal{V}}:\left.\left.(\bar{X}=\overline{\mathrm{t}})\right|_{\bar{X}} \leftrightarrow(\bar{X}=\overline{\mathrm{s}})\right|_{\bar{X}}, \overline{\mathrm{t}}$ ind $\bar{X}$ and $\overline{\mathrm{s}}$ ind $\bar{X}$ (cf. proposition 3 ). In other words, the tuples $\overline{\mathbf{s}}$ and $\overline{\mathrm{t}}$ are members of the same equivalence class of $\bar{\Upsilon}_{\mathbf{I}}$ (cf. def.2). In general the tuple $\overline{\mathbf{s}}$ is not unique and one needs an algorithm for the construction of a solved form wrt a nte-interpretation. The example below illustrates important details.

Example 3. The least fixpoint for the app-predicate of example 2 consists of two quotient flat constraint atoms

$$
\operatorname{app}(X, L, Y) \diamond X=[] \wedge L=Y \text { and } \operatorname{app}(X, L, Y) \diamond X=[H] \wedge Y=[H \mid L]
$$

noted $A_{1} \approx \mathbf{I}$ and $A_{2} \approx \mathbf{I}$, respectively. The atoms $A_{1} \approx \mathbf{I}$ and $A_{2} \approx \mathbf{I}$ represent all possible elements of the $s$-success set of the app-predicate. Recall that the set of the abstract terms $\Upsilon_{\mathbf{I}}$ (the abstract domain) induced by the interpretation $\mathbf{I}$ of example 1 (see section 3 ) is the set $\left\{\tau_{v}, \tau_{\gamma_{c}}, \tau_{\gamma_{f}}, \tau_{\iota}\right\}$ of the equivalence classes of terms. We need the set $\bar{\Upsilon}_{\mathbf{I}}^{(3)}$, which determines the precision of the approximation (one has to construct it).

The properties which we are looking for are groundness, definite freeness and dependency analysis of $\mathcal{O}_{\text {app }}$. This analysis can be done for each element of the least fixpoint separately.

Take the atom $A_{1}$. It is equivalent wrt $\mathbf{I}$ of example 1 with any atom having the form $\left.\operatorname{app}(X, L, Y) \diamond(X=c \wedge L=U \wedge Y=U)\right|_{X, L, Y}$, where $c$ is an arbitrary constant of $\mathcal{L}$ (so the syntactically solved form of $A_{1}$ is not unique). However, for any atom $A^{\prime}$, which is equivalent with $A_{1}$ wrt $\mathbf{I}$ and which has the solved form $\left.\operatorname{app}(X, L, Y) \diamond(\langle X, L, Y\rangle=\overline{\mathrm{t}})\right|_{X, L, Y}$ holds $\left.\overline{\mathrm{t}} \approx_{\mathbf{I}}<\square, U, U\right\rangle$. This gives the following properties: $X$ is always ground, $L$ and $Y$ are free variables and are dependent.

A similar analysis can be performed for the atom $A_{2}$. For any atom $A^{\prime}$, which is equivalent with $A_{2}$ wrt $\mathbf{I}$ and which has the solved form $\operatorname{app}(X, L, Y) \diamond(<$ $X, L, Y>=\overline{\mathrm{t}})\left.\right|_{X, L, Y}$, holds: $\overline{\mathrm{t}} \approx \mathbf{I}<\left[U_{1}\right], U_{2},\left[U_{1} \mid U_{2}\right]>$. This completes the analysis of the atom $A_{2}$. It shows that $X$ and $Y$ are dependent, also $Y$ and $L$ are dependent. $X$ and $L$ are independent; $L$ is free but $X$ and $Y$ are not. Formally, the result just above has to be justified by the analysis of the equivalence classes of tuples of terms of $\bar{\Upsilon}_{\mathbf{I}}$. In this particular case it is not difficult.

Now the above results of the analysis of the atoms $A_{1}$ and $A_{2}$ can be combined to obtain the characterisation of the $s$-success set of the app-predicate. The argument $L$ is always free, $X$ and $Y$ are not free, $L$ and $Y$ are always dependent,.... This completes the groundness, freeness and dependency analysis of $\mathcal{O}_{a p p}$. At this step some information is lost, because the separate analysis has shown that $\mathcal{O}_{\text {app }}$ can consists of two disjoint parts having different properties. 
The above schema for extracting properties may produce unsafe results. Consider for instance a program with the following two clauses: $p(X) \leftarrow \diamond X=U$ and $p(X) \diamond[b, X]=[U, a]]$. The first clause contributes $[p(X) \leftarrow \diamond X=U]^{\approx} \mathbf{I}$. The second clause contributes $[p(X) \diamond[b, X]=[U, a]]^{\approx} \mathbf{I}$ wrt I of example 1 . This atom is equivalent with the former, so it could be removed from the fixpoint. However, doing so yields an unsafe analysis, as both clauses produce answers which are different regarding the properties of groundness and freeness.

As shown in [2], the problem can be solved with the following definition ${ }^{5}$. A constraint atom having the form $\left.p(\bar{X}) \diamond \mathbf{E}\right|_{\bar{X}}$ is said to be positive wrt $\mathbf{I}$ iff $\left.\mathbf{E}\right|_{\bar{X}}$ has a solved form which is obtainable using only $E Q$-axioms and if it is derived by applying the $\mathbf{T}_{\boldsymbol{P}}$ operator on positive atoms. An atom, which does not satisfy this condition, is said to be negative. In the context of example 2 both app-atoms are positive; the $p$-atom is negative because its atomic constraint has no solved form. In the example program just above, the atom in the fixpoint originating from the first clause is positive, that of the second is negative.

A negative atom only contains negative information concerning the properties of computed answer substitutions. For instance, the negative $p$-atom in the context of example 2 shows that: if $Y$ is ground then $L$ is ground; $Y$ is not free, i.e. computed answer substitutions with ground $Y$ and non-ground $L$ or with free $Y$ do not belong to the $s$-success set of $p$.

It can be shown that the analysis produces always safe results if (1) a negative atom is removed from the fixpoint if it is equivalent with a negative atom already in the fixpoint, and (2) a positive atom is only removed if it is equivalent with another positive atom already in the fixpoint set.

The analysis of $a p p$-predicate in example 3 is safe because the app-atoms obtained in the third and fourth iteration are positive and equivalent with a positive atom already in the fixpoint, so they can be removed.

Let us summarise the basic steps which are necessary in our framework. The first step is the abstract domain construction, which requires a precision theory as input (section 3 ). The precision should be chosen in advance to fit the particular problem at hand. The second step is a least fixpoint computation. It uses the preinterpretation obtained as a result of the abstract domain construction. Finally, the analysis of the least fixpoint derives properties of the program.

The first and the last step are mutually dependent, because the precision should fit the set of properties which are of interest in the analysis step. This is a problem which is not addressed in this paper. An approach to this problem relies on controlling the properties of the abstract domains $\bar{\Upsilon}_{\mathbf{I}}^{(k)}, k=1,2, \ldots$ by varying the nte-interpretation I. However, the above example has shown some intuition: the equivalence classes (abstract tuples of terms) have been chosen to fit the requirements of freeness and groundness analysis (the above dependency analysis is a kind of "side effect"). This choice was rather obvious due the fact that the problem is well-known.

${ }_{5}^{5}$ The definition uses an observation suggested by John Shepherdson. 


\section{Conclusion}

We have presented a novel approach for abstract interpretation of definite logic programs. The advantages have been illustrated using the bottom-up variant of the $s$-semantics. The choice has been motivated by the possibility of a simple and elegant formalisation. However, the proposed schema based upon "abstracting" non-term equality theories can be adapted to all all settings, which are based on the classical notion of a most general unifier such as SLD-resolution (see, for example, [4]).

The recent paper [5] is closely related and was a strong motivation for developing our approach. The advantage of our setting is that the syntactical notion of most general unifier has received a model-theoretic explanation on the basis of non-term equality theories. This immediately has given an important outcome. One obtains a flexible abstract domain construction technique, which offers an alternative to the idea of combining domains [9, 10]. The latter has shown that combining abstract domains enables to increase precision and efficiency of an analysis. We hope that precision theories can be used to specify abstract domains, which fit problems exactly and, therefore, gives rise to efficient implementation. The domain generation by means of a model generator is computationally very hard (sometimes a complete search), but this should be done as a part of compiler design, i.e. in a program independent way.

The selection of precision theories, avoiding negative elements in the fixpoint, is a topic of further research. We expect that a special refinement technique can be elaborated, which allows to derive very complex properties (described as abstract data types) of definite logic programs. The latter may give rise to an approach for compiling definite logic programs into efficient imperative programs.

An important issue is the possibility of efficient implementation. The recent paper [16] reports very nice results concerning an efficient implementation of a bottom-up analysis aimed at deriving complex properties of definite logic programs. We hope that the elaboration of the "theoretical" schema of our variant of the bottom-up computations will fit the setting of [16].

An interesting application exploiting a special pre-interpretation (an abstract domain), which is constructed as a model of the precision theory describing the requirements of the compiling control problem, can be found in [3].

\section{Acknowledgements}

We are grateful to Bart Demoen and Gerda Janssens for fruitful discussions.

\section{References}

1. Bancilhon,F., Ramakrishnan,R., An Amateur's Introduction to Recursive Query Processing Strategies, Proc. ACM-SIGMOD Conf., 1986, 16-52.

2. Boulanger,D., Bruynooghe,M., A Systematic Construction of Abstract Domains, Draft, June, 1994. 
3. Boulanger,D., De Schreye,D., Compiling Control Revisited: A New Approach based upon Abstract Interpretation, Proc. 11-th Int. Conf. Logic Programming, 1994.

4. Bruynooghe,M., Boulanger,D., Abstract Interpretation for (Constraint) Logic Programming, in: Constraint Programming, Eds. B.Mayoh, E.Tõugu, J.Penjam, NATO Adv. Science Series, Comp. and System Sci., Springer, 1994. (to appear)

5. Bruynooghe,M., Codish,M., Freeness, Sharing, Linearity and Correctness - All at Once, Proc. 3-rd Int. Workshop WSA'93, Springer-Verlag, 1993.

6. Bry,F., Decker,H., Manthey,R., A Uniform Approach to Constraint Satisfaction and Constraint Satisfiability in Deductive Databases, Proc. Extended Database Technology 1988, Venice, LNCS, Springer, 1988.

7. Bossi, A., Gabrielli, M., Levi G., Martelli, M., The 3-semantics Approach: Theory and Applications, (to appear in J. Logic Programming).

8. Codish,M., Demoen,B., Analysing Logic Programs using Prop-ositional Logic Programs and a Magic Wand, Proc. 1993 Int. Symp. on Logic Prog., 1993, 114-129.

9. Codish,M., Mulkers,A., Bruynooghe,M., Garcia de la Banda,M., Hermenegildo,M., Improving Abstract interpretations by Combining Domains, Proc. ACM Symp. on Partial Evaluation and Semantics based Program Manipulation, 1993.

10. Cousot,P., Cousot,R., Systematic Design of Program Analysis Frameworks, Proc. 6-th ACM Symp. Principles Prog. Languages, San Antonio, USA, 1979, 269-282.

11. Codognet,P., File, G., Computations, Abstractions and Constraints in Logic Programs, Proc. 4-th Int. Conf. Programming Languages, Oakland, USA, 1992.

12. Denecker,M., De Schreye,D., A Framework for Indeterministic Model Generation with Equality, Report CW124, K.U.Leuven, 1992.

13. Denecker,M., Knowledge Representation and Reasoning in Incomplete Logic Programming, Ph.D. Thesis, Dept. Comp. Science, K.U.Leuven, 1993.

14. Falaschi,M., Levi,G., Martelli,M., Palamidessi,C., Declarative Modelling of the Operational Behaviour of Logic Languages, Theoretical Computer Science, 1989, Vol.69, No.3, 289-318.

15. Frühwirth,T., Shapiro,E., Vardi,M., Yardeni,E., Logic Programs as Types for Logic Programs, Proc. 6-th IEEE Symp. Logic in Comp. Sci., Amsterdam, July 1991.

16. Gallagher,J., de Waal,D.A., Fast and Precise Regular Approximations of Logic Programs, Proc. 11-th Int. Conf. Logic Programming, 1994.

17. Giacobazzi,R., Debray,S., Levi,G., Generalised Semantics and Abstract Interpretation for Constraint Logic Programs, Proc. 5-th Int. Conf. FGCS, Tokyo, 1992.

18. Janssens, G., Bruynooghe,M., Deriving Descriptions of Possible Values of Program Variables by Means of Abstract Interpretation, J, Logic Programming, 1992, Vol.13, Nos.2-3, 205-258.

19. Lassez,J.-L., Maher,M., Mariott,K., Unification Revisited, Foundations of Deductive Databases and Logic Programming, ed. J.Minker, Morgan-Kaufmann, 1988, 587-625.

20. Lloyd,L., Foundations of Logic Programming, Springer, Berlin, 1987.

21. Martelli,A., Montanari,U., An Efficient Unification Algorithm, Transaction on Programming Languages and Systems, 1982, Vol.2, No.4, 258-282.

22. Shepherdson,J., Language and Equality Theory in Logic Programming, Report No.PM-91-02, School of Mathematics, Bristol, England. 